OPEN ACCESS

Edited by:

Fan Jin,

Zhejiang University, China

Reviewed by:

Yue-qiu Tan,

Central South University, China

Chengliang Zhou,

International Peace Maternity and

Child Health Hospital, China

*Correspondence:

Yanmin Luo

luoyanm@mail.sysu.edu.cn

Yi Zhou

zhouyi6@mail.sysu.edu.cn

Specialty section

This article was submitted to

Genetics of Common and Rare

Diseases,

a section of the journa

Frontiers in Genetics

Received: 13 December 2020 Accepted: 15 February 2021

Published: 04 March 2021

Citation:

Lin S, He Z, Huang L, Liu J, Lei T,

Wu J, Huang $P$, Zhou $Y$ and Luo $Y$ (2021) Case Report: Low-Level

Maternal Mosaicism of a Novel CREBBP Variant Causes Recurrent Rubinstein-Taybi Syndrome in Two

Siblings of a Chinese Family.

Front. Genet. 12:640992.

doi: 10.3389/fgene.2021.640992

\section{Case Report: Low-Level Maternal Mosaicism of a Novel CREBBP Variant Causes Recurrent Rubinstein-Taybi Syndrome in Two Siblings of a Chinese Family}

\author{
Shaobin Lin ${ }^{1}$, Zhiming He ${ }^{1}$, Linhuan Huang ${ }^{1}$, Jialiu Liu ${ }^{1}$, Ting Lei ${ }^{2}$, Jianzhu Wu ${ }^{1}$, \\ Peizhi Huang ${ }^{1}$, Yi Zhou ${ }^{1 *}$ and Yanmin Luo ${ }^{1 *}$ \\ ${ }^{1}$ Department of Obstetrics and Gynecology, The First Affiliated Hospital of Sun Yat-sen University, Guangzhou, China, \\ ${ }^{2}$ Department of Ultrasonic Medicine, The First Affiliated Hospital of Sun Yat-sen University, Guangzhou, China
}

Familial Rubinstein-Taybi syndrome (RSTS) with recurrent RSTS siblings and apparently unaffected parents is rare; such cases might result from parental somatic and/or germline mosaicism. Parental low-level $(<10 \%)$ germline mosaicism in the CREBBP-associated RSTS family has not been reported. Here, we present our studies of a Chinese family with two RSTS siblings and apparently unaffected parents. We detected the apparent de novo variant (DNV) c.3235C>T (p.Gln1079*) in CREBBP in the siblings via trio whole-exome sequencing. High-depth next-generation sequencing (NGS) for the parents revealed a low-level $(<10 \%)$ mosaic variant in both the peripheral blood $(3.64 \%)$ and buccal mucosa $(1.94 \%)$ of the unaffected mother, indicating maternal somatic and germline mosaicism. Peripheral blood RNA-sequencing analysis for the patients and normal individuals indicated that the c.3235C > T (p.Gln1079*) non-sense variant did not trigger nonsense-mediated mRNA decay to reduce CREBBP mRNA levels. Transcriptome analysis revealed 151 downregulated mRNAs and 132 upregulated mRNAs between the patients and normal individuals. This study emphasizes that high-depth NGS using multiple specimens might be applied for a family with an affected sibling caused by an apparent CREBBP DNV to identify potential low-level parental mosaicism and provide an assessment of recurrence risk.

Keywords: CREBBP, germline mosaicism, Rubinstein-Taybi syndrome, next-generation sequencing, RNA sequencing

\section{INTRODUCTION}

Rubinstein-Taybi syndrome (RSTS) is a rare genetic syndrome featuring distinctive facial dysmorphisms, characteristic hand and foot findings, short stature, developmental delay, and intellectual disability, with a reported incidence of 1:100,000-1:125,000 births in the Netherlands (Hennekam et al., 1990; Wiley et al., 2003; Hennekam, 2006). CREBBP gene variants, including single-nucleotide variants and exonic or whole-gene deletions, are reported to be the cause of RSTS type 1 (RSTS1; OMIM \#180849), with autosomal dominant inheritance, contributing to $\sim 50-60 \%$ of RSTS cases (Korzus, 2017). 
In families with clinically unaffected parents, CREBBP variants are considered to mostly derive from a de novo event (Bartsch et al., 2010). Although the recurrence risk in RSTS families is generally regarded to be low, either an unrecognized mild phenotype in a parent with heterozygosity (or somatic mosaicism) or unidentified germline mosaicism in an unaffected parent is presumed to contribute to a risk of RSTS recurrence in their offspring. The recurrence risk of a family with an affected sibling caused by an apparently de novo variant (DNV) is estimated to range from 0.048 to $9.4 \%$ (Campbell et al., 2014).

Overall, the detection of low-level mosaicism where the percent of cells with the variant are $<10 \%$ with variant allele fractions (VAFs) $<10 \%$ is challenging, while the detection of high-level mosaicism where the percent of cells with the variant are more than $10 \%$ with VAFs $\geq 10 \%$ is currently expected to be feasible (Gambin et al., 2020; Rodríguez-Martín et al., 2020). Nevertheless, recent advances in high-sensitivity genetic tests, including high-depth next-generation sequencing (NGS) and droplet digital PCR (ddPCR), have allowed for the more precise detection of low-level mosaicism that might be missed by traditional Sanger sequencing or low-depth NGS (Cao et al., 2019; Hu et al., 2019). In addition, it has been reported that routine exome sequencing (ES) variant calling pipelines are unable to detect variants with VAFs lower than 10\% (Gambin et al., 2020). Therefore, it might be questioned whether some of the previously reported, presumed DNVs in probands might derive from parents with unidentified low-level somatic and/or germline mosaicism (Jonsson et al., 2018; Hu et al., 2019; Gambin et al., 2020). Here, we present our studies of a Chinese family with two boys with RSTS and apparently unaffected parents. With the use of trio whole-exome sequencing (WES), we detected a novel CREBBP variant in the two children; subsequent high-depth NGS identified a mosaic variant in the mother, indicating low-level maternal germline mosaicism $(<10 \%)$ for the first time.

\section{CASE PRESENTATION}

Two male children presented to our genetics clinic at the ages of 13 and 16 years. They were born to a non-consanguineous family, and abnormal pregnancy or delivery events were not noted. According to their medical records and descriptions, the parents had no recognizable phenotype or significant medical conditions. At the time that the two children were referred for clinical genetic assessment, the height of the older and younger brother was $152 \mathrm{~cm}(<3 \mathrm{rd}$ percentile) and $130 \mathrm{~cm}(<3 \mathrm{rd}$ percentile), and their weights were $53 \mathrm{~kg}$ (25th percentile) and $40 \mathrm{~kg}$ (19th percentile), respectively. The boys were able to walk alone at the ages 25 and 28 months and spoke their first words at 23 and 25 months, respectively. They showed common clinical features, including the characteristic facial appearance, short stature, speech and motor delay, intellectual disability, microcephaly, hearing impairment, glaucoma, crowding of teeth and malocclusion, broad and angulated thumbs and halluces, bilateral syndactyly between the great and second toe, and cryptorchidism (Figure 1). The characteristic facial appearance includes marked eyebrows, downslanted palpebral fissures, broad and prominent nose, low-hanging columella, strabismus, and micrognathia (Figure 1). Behavior problems were observed, with aggressive behaviors, distractibility, instability of mood, and stereotypies. Other medical conditions included recurrent upper respiratory infections, gastroesophageal reflux, and constipation. The parents provided informed consent for this research and publication of the data.

\section{METHODS}

\section{Karyotyping and Chromosomal Microarray Analysis}

Traditional G-banded karyotyping was performed on peripheral blood using standard protocols. CMA was conducted using a single-nucleotide polymorphism (SNP) array platform with Affymetrix CytoScan HD arrays (Thermo Fisher Scientific, Inc., Waltham, Massachusetts, USA) according to the manufacturer's protocols.

\section{WES}

Exome sequences were captured by NimbleGen SeqCap EZ Exome v3 (Roche NimbleGen, Madison, Wisconsin, USA); after enrichment and purification, the exome libraries were sequenced using the NextSeq500 platform (Illumina, San Diego, CA, USA). Sequence reads were aligned to the February 2009 human reference sequence (GRCh37/hg19) assembly. The sequencing process, variant filtration and interpretation followed the protocols in a previous study (Fu et al., 2020).

\section{Sanger Sequencing}

PCR and Sanger sequencing were performed using standard methods. The primer sequences used for PCR and sequencing are available upon request.

\section{PCR Amplicon-Based High-Depth NGS}

PCR amplicon-based NGS was performed as previously described (Gambin et al., 2020). Briefly, parental genomic DNA from peripheral blood, sperm, and buccal mucosa was amplified using primers targeting putative mosaic variants. The PCR products were purified, and the purified amplicons were sequenced using the Illumina NovaSeq 6000 platform (Illumina, San Diego, CA, USA).

\section{RNA Sequencing}

Total RNA was extracted from peripheral blood using TRIzol (Thermo Fisher Scientific, Inc., Waltham, Massachusetts, USA). RNA-Seq libraries were prepared using $3 \mu \mathrm{g}$ of total RNA. An Epicenter Ribo-Zero ${ }^{\mathrm{TM}}$ rRNA Removal Kit (Epicenter, USA) was employed to remove ribosomal RNA and a Directional RNA Library Prep Kit (NEB, USA) to prepare the sequencing libraries. The Illumina HiSeq 4000 (Illumina, San Diego, CA, USA) was utilized for RNA sequencing. The RNA sequencing procedure, data processing and analysis were in accordance with a previous study (Fu et al., 2020). 


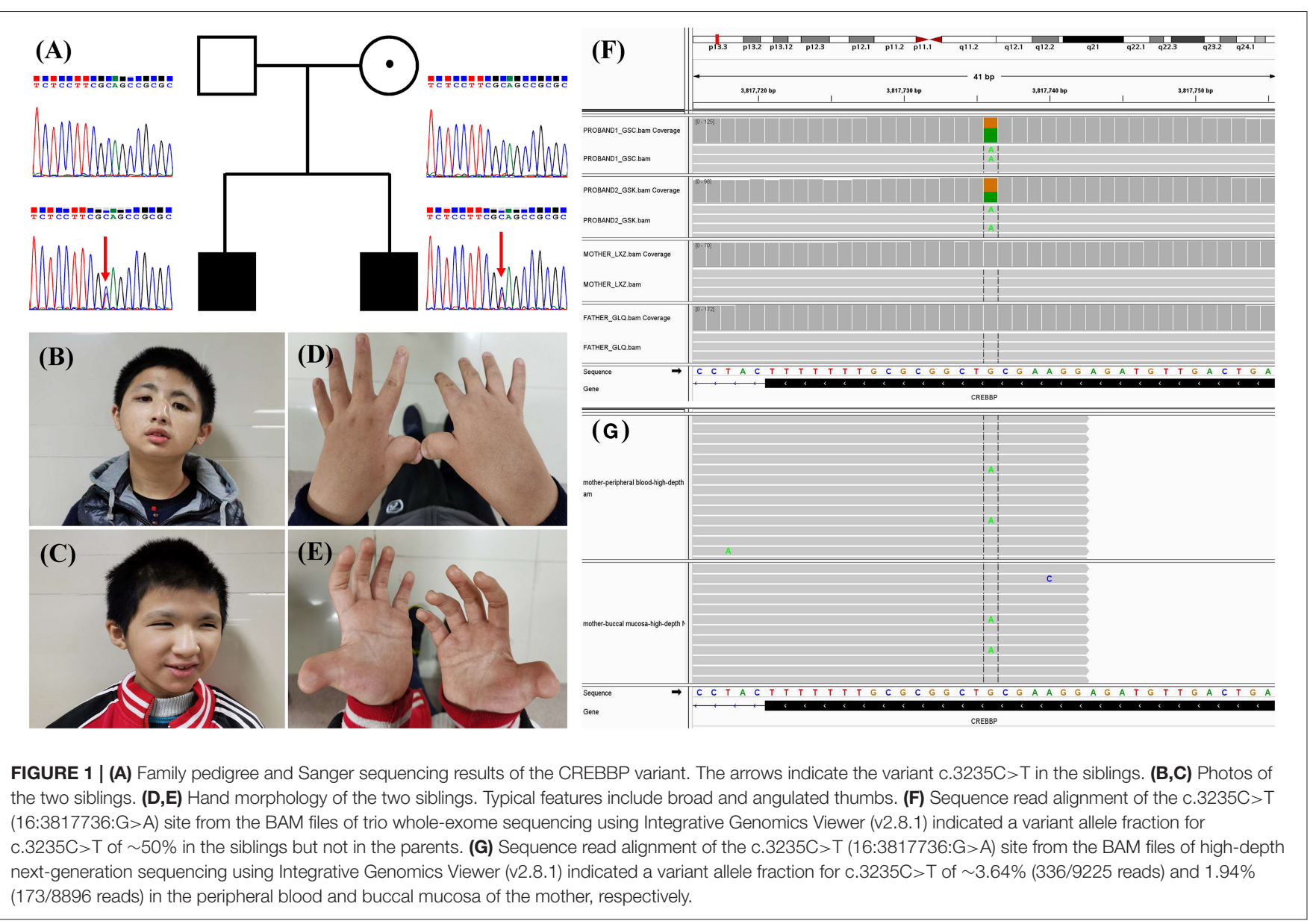

\section{RESULTS}

\section{Karyotyping and CMA}

G-banded karyotyping and CMA did not reveal relevant chromosomal abnormalities, copy number variants $(>100 \mathrm{~kb})$ or runs of homozygosity ( $>5 \mathrm{Mb}$ ) in the two siblings.

\section{WES, Sanger Sequencing, and Amplicon-Based High-Depth NGS}

The mean coverage depth for the WES data was $\geq 160 \times$, with more than $99.8 \%$ of the target regions covered at $\geq 20 \times$. Through a gene filtration and bioinformatic analysis process, a de novo nonsense variant 16:3817736:G > A, c.3235C > T (p.Gln1079*), in exon 16 of CREBBP (NM_004380.3) was identified in the two children (Figure 1). The variant is absent in public population genomic databases. The variant is classified as likely pathogenic according to American College of Medical Genetics (ACMG) variant interpretation guidelines.

Intriguingly, WES did not reveal the variant in the unaffected parents (Figure 1). Moreover, Sanger sequencing confirmed the heterozygous c.3235C > T (p.Q1079*) variant in the two affected children but not in the parents (Figure 1). We then employed PCR amplicon-based high-depth NGS to investigate a putative mosaic variant in one of the parents and ultimately identified mosaic c.3235C > T (p.Q1079*) variants in the peripheral blood (3.64\%, alternate reads/total reads $=336 / 9225)$ and buccal mucosa $(1.94 \%$, alternate reads/total reads $=173 / 8896)$ of the mother but not the father (Figure 1).

\section{RNA Sequencing}

We then investigated whether the $c .3235 \mathrm{C}>\mathrm{T}$ variant influences CREBBP mRNA levels using RNA-sequencing analysis of peripheral blood from the patients and normal individuals. However, the level of CREBBP mRNA was not significantly different between the patients and normal individuals [log2 ratio (patients/controls) $=-0.0032274829851164$; $Q$-value $=0.996318464271172]$.

Transcriptome analysis was then applied to compare peripheral blood genome-wide expression profiles between the patients and normal individuals. In total, 283 genes showed significantly differential expression between them. Among these, 151 mRNAs were downregulated, and another 132 mRNAs were upregulated. The top 20 genes with significantly differential mRNA expression are listed in Table 1, of which 10 were upregulated and the other 10 downregulated. Furthermore, we performed Gene Ontology (GO) and Kyoto Encyclopedia of Genes and Genomes (KEGG) analyses for all differentially expressed mRNAs induced by the CREBBP c.3235C $>$ T variant. 
TABLE 1 | Top 10 upregulated and 10 downregulated mRNAs between the patients and normal individuals.

\begin{tabular}{|c|c|c|c|c|c|c|c|c|}
\hline $\begin{array}{l}\text { Gene } \\
\text { symbol }\end{array}$ & $\begin{array}{c}\text { Patients } \\
\text { expression }\end{array}$ & $\begin{array}{c}\text { Normal } \\
\text { individuals } \\
\text { expression }\end{array}$ & $\begin{array}{l}\text { Patients } \\
\text { average } \\
\text { read count }\end{array}$ & $\begin{array}{l}\text { Normal } \\
\text { individuals } \\
\text { average } \\
\text { read count }\end{array}$ & log2 ratio & $\begin{array}{l}\text { Up/down } \\
\text { regulation }\end{array}$ & $P$-value & $Q$-value \\
\hline HLA-DRB5 & 225.835 & 0.765 & 3532.39 & 13.875 & 7.482495967 & up & 4.91E-55 & 1.78E-52 \\
\hline MYBL1 & 26.42 & 0.65 & 1820 & 51.5 & 4.544114327 & up & $8.29 E-42$ & 2.47E-39 \\
\hline MYOM2 & 38.7 & 1.05 & 2678.5 & 78.5 & 4.529897009 & up & 1.13E-35 & 2.32E-33 \\
\hline FOSB & 19.76 & 0.52 & 1006 & 29.5 & 4.506423885 & up & 2.03E-09 & 3.04E-08 \\
\hline KLRF1 & 68.75 & 2.09 & 1035 & 36.5 & 4.259903293 & up & $5.50 \mathrm{E}-25$ & $6.66 \mathrm{E}-23$ \\
\hline CXCL8 & 42.075 & 1.37 & 890 & 32.5 & 4.218315485 & up & $1.28 \mathrm{E}-11$ & $3.02 \mathrm{E}-10$ \\
\hline GoS2 & 50.5 & 1.29 & 574 & 20 & 4.161368474 & up & 1.36E-08 & 1.63E-07 \\
\hline RGS1 & 11.715 & 0.375 & 207 & 8 & 4.086850386 & up & $6.25 \mathrm{E}-10$ & 1.05E-08 \\
\hline RPL36A & 262.95 & 54.965 & 1548.5 & 422 & 4.083172057 & up & $1.11 \mathrm{E}-12$ & $3.43 \mathrm{E}-11$ \\
\hline RPL34 & 1522.475 & 59.685 & 9236 & 422 & 3.965364098 & up & $1.23 \mathrm{E}-11$ & $2.91 \mathrm{E}-10$ \\
\hline ALAS2 & 1.745 & 878.28 & 45.5 & 26335.5 & -9.768604841 & down & 1.24E-212 & 4.07E-209 \\
\hline SLC4A1 & 0.455 & 82.065 & 22.5 & 6477 & -8.813880937 & down & $4.48 \mathrm{E}-90$ & $2.93 \mathrm{E}-87$ \\
\hline $\mathrm{IFI} 27$ & 0.94 & 68.11 & 6 & 535 & -7.096128608 & down & $2.88 \mathrm{E}-38$ & 7.25E-36 \\
\hline SLC6A8 & 0.545 & 16.94 & 23.5 & 984.5 & -6.056603232 & down & 4.39E-37 & $1.02 \mathrm{E}-34$ \\
\hline PHOSPHO1 & 6.32 & 128.8 & 171 & 4585 & -5.432012286 & down & $2.61 \mathrm{E}-17$ & $1.53 \mathrm{E}-15$ \\
\hline SLC25A39 & 44.395 & 819.91 & 1020.5 & 21348 & -5.05753814 & down & $3.05 E-36$ & 6.64E-34 \\
\hline PDZK1IP1 & 1.49 & 19.975 & 15.5 & 291 & -4.951911648 & down & $5.28 \mathrm{E}-11$ & 1.11E-09 \\
\hline NLRP6 & 0.835 & 13.215 & 32.5 & 565.5 & -4.679911744 & down & $1.89 \mathrm{E}-27$ & $2.95 E-25$ \\
\hline MMP9 & 3.01 & 31.465 & 93 & 1361.5 & -4.630504037 & down & 0.006132 & 0.009425 \\
\hline ALPL & 5.28 & 64.135 & 182.5 & 2705.5 & -4.580305046 & down & $9.21 \mathrm{E}-14$ & 3.34E-12 \\
\hline
\end{tabular}

GO analysis revealed these mRNAs to be mainly enriched in transmembrane signaling receptor activity and inflammatory response terms. Based on the KEGG analysis, these mRNAs are mostly associated with pathway in the cytokine-cytokine receptor interaction (Figure 2).

\section{DISCUSSION}

To date, the majority of CREBBP-associated RSTS are sporadic cases caused by DNVs. Familial CREBBP-associated RSTS in a parent-to-child transmission manner or a recurrent condition with multiple affected siblings and apparently unaffected parents in a family might result from parental somatic and/or germline mosaicism. Indeed, CREBBP mosaic variants have been previously reported in eight RSTS cases (Table 2) (Gervasini et al., 2007; Chiang et al., 2009; Bartsch et al., 2010; Tajir et al., 2013; De Vries et al., 2016). In the study from Gervasini et al. (2007), high-level (ranging from 17 to 30\%) somatic mosaicisms of CREBBP partial deletions were identified via fluorescence in situ hybridization in three cases with RSTS. De Vries et al. (2016) also reported a somatic mosaicism of CREBBP c.5039_5041delCCT (p.Ser1680del) in the buccal mucosa but not the peripheral blood from a patient with RSTS and Filippi syndrome. However, whether germline mosaicisms were also present in these cases in the two studies remained unknown. To our knowledge, parental germline mosaicism of CREBBP was assumed in four families (Chiang et al., 2009; Bartsch et al., 2010; Tajir et al., 2013). All of them showed high-level mosaic variants and were expected to be easily detected by Sanger sequencing or pyrosequencing. However, we noted that there was sufficient evidence supporting an assumed parental germline mosaicism in the family reported by Bartsch et al. (2010), in which the mosaic c.4134G $>$ T (p.Arg $>$ Arg) was detected by Sanger sequencing or pyrosequencing in the peripheral blood and buccal mucosa from the proband's father. Additionally, there was no sufficient evidence supporting a confirmation of parental germline mosaicism in the three families reported by Chiang et al. (2009) and Tajir et al. (2013), in which the mosaic CREBBP variants were not revealed by Sanger sequencing in the peripheral blood and buccal mucosa from the proband's parents. Of note, low-level parental germline mosaicism could still not be excluded by Sanger sequencing or pyrosequencing without high-sensitivity performance on the detection of mosaicism. Actually, many reports do not provide direct evidence for germline mosaicism because direct identification of germline mosaicism for females is difficult (possibly invasive ovarian tissue collection), though it is feasible for males (sperm).

Regardless, high-depth NGS and ddPCR have enabled assessing somatic and/or germline mosaicism in families with DNVs (Hu et al., 2019). Here, we report a novel heterozygous CREBBP variant in two siblings with unique clinical features in a Chinese family. The phenotypes of the two siblings are highly consistent with classical RSTS. Because routine trioWES analysis did not identify the variant in the unaffected parents, parental low-level germline mosaicism was suspected and then confirmed by high-depth NGS. In this study, we 


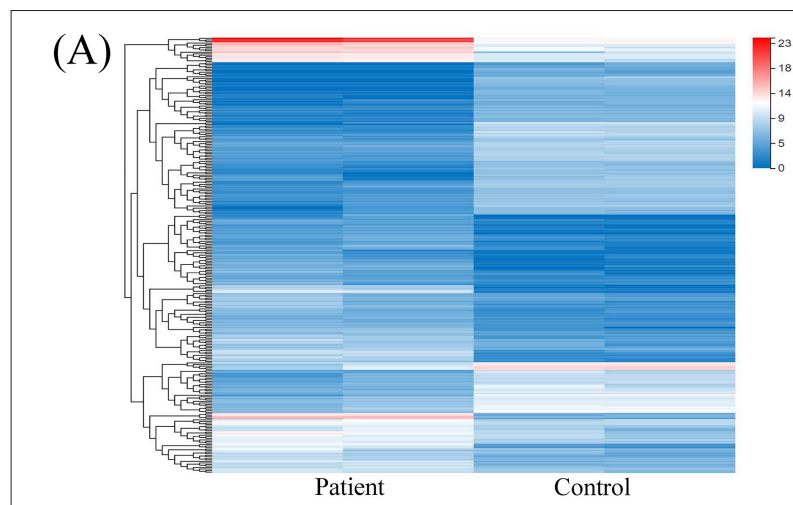

(C)

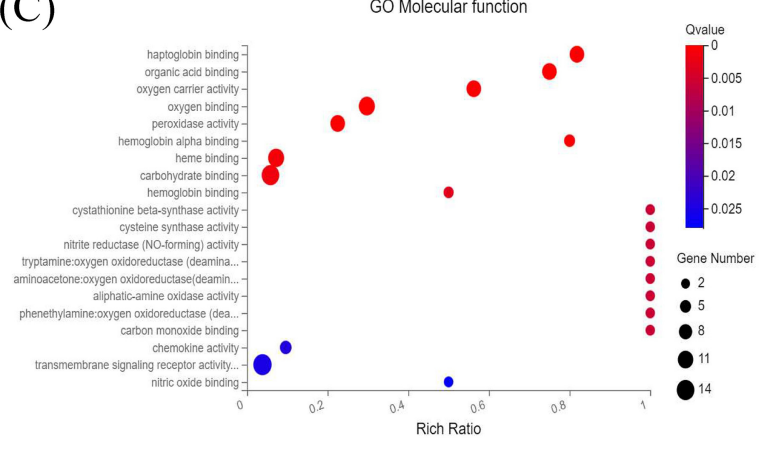

(B)

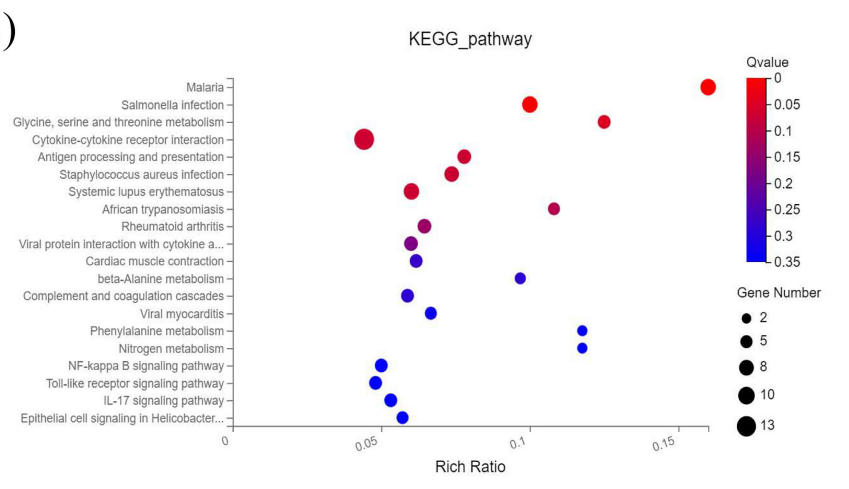

(D)

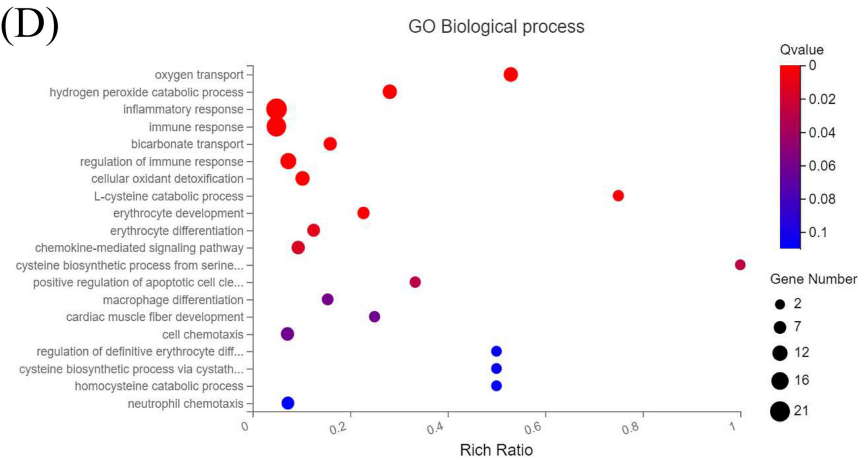

FIGURE 2 | RNA sequencing analysis of the peripheral blood from the patients and normal individuals and functional annotation of mRNAs. (A) Heat map of mRNAs with differential expression in peripheral blood from the patients and normal individuals. (B) KEGG pathway analysis of mRNAs with differential expression. (C,D) GO enrichment analysis of mRNAs with differential expression.

used multiple specimens, including peripheral blood, sperm and buccal mucosa, to investigate c.3235C $>$ T (p.Gln1079*) mosaicism in the parents. Consequently, the mother was revealed to be a carrier of a c.3235C > T (p.Gln1079*) low-level mosaic variant in the peripheral blood (3.64\%) and buccal mucosa (1.94\%), indicating maternal somatic and germline mosaicism.

To date, 209 unique pathogenic or likely pathogenic variants of CREBBP have been recorded in the Leiden Open Variation Database (http://www.LOVD.nl/CREBBP). Approximately 50\% of pathogenic variants related to CREBBP-associated RSTS cluster in chromatin-modifying regions (HAT and Bromo domains) in the CREBBP gene, especially the former (Korzus, 2017). A previous study demonstrated that the sole loss of HAT activity was sufficient to cause RSTS (Kalkhoven et al., 2003). The c.3235C $>$ T (p. Gln1079*) variant is located in exon 16 and maps to the region between the KIX and Bromo domains. Very few variants in exon 16 are reported to contribute to RSTS (Korzus, 2017). In fact, only one, c.3096dup (p.Lys1033*), is located in exon 16 according to the Leiden Open Variation Database. The c.3235C > T (p.Gln1079*) variant is predicted to produce a truncated protein consisting of 1079 amino acids excluding the Bromo, HAT, and TAZ2 domains. Loss of these critical domains might affect the wild-type protein and might lead to haploinsufficiency to cause RSTS, which is also supported by the observation that the two siblings had consistent phenotypes matching classic RSTS.
CREBBP transcription produces an $\sim 10 \mathrm{~kb}$ mRNA containing a $7.3 \mathrm{~kb}$ coding sequence. CREBBP is extensively expressed in various tissues in humans, including peripheral blood mononuclear cells. RNA sequencing of peripheral blood from the patients and normal individuals demonstrated no difference in mRNA levels between them. Thus, c.3235C> T (p.Gln1079*) mutant transcripts might be stable, with transcription levels similar to those of the wild-type transcripts, and the c.3235C $>\mathrm{T}$ (p.Gln1079*) variant in exon 16 of CREBBP might escape nonsense-mediated mRNA decay. We also established peripheral blood mRNA profiles for the patients and normal individuals and detected 151 downregulated and 132 upregulated mRNAs between them. GO and KEGG pathway analyses revealed that these mRNAs are enriched in transmembrane signaling receptor activity and inflammatory response terms and pathway in the cytokine-cytokine receptor interaction. Overall, further research should be performed to investigate whether the c.3235C $>\mathrm{T}$ (p.Gln1079*) variant contributes to RSTS via these pathways.

Parental somatic and/or germline mosaicism causing recurrent autosomal dominant or X-linked genetic disorders in a family are rare but not uncommon (Smith et al., 1999; Aarabi et al., 2018; Hu et al., 2019; Liu et al., 2019). To our knowledge, parental low-level $(<10 \%)$ somatic and germline mosaicism in a CREBBP-associated RSTS family has not been reported with sufficient evidence. Together with many previous studies and given the recurrent probability of a family with an affected sibling caused by an apparent DNV in CREBBP, we suggest that 
TABLE 2 | Summary of previously reported cases with mosaic variants in CREBBP.

\begin{tabular}{|c|c|c|c|c|c|c|c|c|c|}
\hline Study & Case & $\begin{array}{l}\text { CREBBP variant in } \\
\text { the proband }\end{array}$ & $\begin{array}{l}\text { CREBBP variant } \\
\text { in one of the } \\
\text { parents }\end{array}$ & Mosaic ratio & $\begin{array}{l}\text { Detection } \\
\text { method }\end{array}$ & $\begin{array}{l}\text { Somatic } \\
\text { mosaicism }\end{array}$ & $\begin{array}{l}\text { Germline } \\
\text { mosaicism }\end{array}$ & $\begin{array}{l}\text { Clinical findings } \\
\text { in cases with } \\
\text { (assumed) } \\
\text { mosaicism }\end{array}$ & $\begin{array}{l}\text { Familial or } \\
\text { recurrent RSTS }\end{array}$ \\
\hline \multirow[t]{3}{*}{$\begin{array}{l}\text { Gervasini et al. } \\
(2007)\end{array}$} & case 38 & mos deletion & unk & $\begin{array}{l}\text { peripheral blood (30\%) } \\
\text { buccal mucosa (unk) }\end{array}$ & FISH & + & unk & RSTS & unk \\
\hline & case 40 & mos deletion & unk & $\begin{array}{l}\text { peripheral blood (20\%) } \\
\text { buccal mucosa (17\%) }\end{array}$ & $\mathrm{FISH}$ & + & unk & RSTS & unk \\
\hline & case 66 & mos deletion & unk & $\begin{array}{l}\text { peripheral blood }(22 \%) \\
\text { buccal mucosa }(21 \%)\end{array}$ & FISH & + & unk & RSTS & unk \\
\hline \multirow[t]{2}{*}{$\begin{array}{l}\text { Chiang et al. } \\
\text { (2009) }\end{array}$} & $\begin{array}{l}\text { family } 1 \text { - one of } \\
\text { the parents }\end{array}$ & 6122-6125delCCAT & - & $\begin{array}{l}\text { peripheral blood (0) buccal } \\
\text { mucosa (0) }\end{array}$ & Sanger & - & assumed & $\begin{array}{l}\text { broad great toes in } \\
\text { the father }\end{array}$ & + \\
\hline & family 2-father & $\begin{array}{l}c .4078 C>T \\
\left(p . \operatorname{Arg} 1360^{*}\right)\end{array}$ & $\begin{array}{l}\text { mos c. } 4078 \mathrm{C}>\mathrm{T} \\
\left(\mathrm{p} . \operatorname{Arg} 1360^{*}\right)\end{array}$ & $\begin{array}{l}\text { peripheral blood (ND) } \\
\text { buccal mucosa (ND) }\end{array}$ & Sanger & ND & assumed & broad great toes & - \\
\hline $\begin{array}{l}\text { Bartsch et al. } \\
\text { (2010) }\end{array}$ & family 2-father & $\begin{array}{l}\text { c. } 4134 \mathrm{G}>\mathrm{T} \\
\text { (p.Arg }>\text { Arg })\end{array}$ & $\begin{array}{l}\text { mos c. } 4134 \mathrm{G}>\mathrm{T} \\
(\mathrm{p} . \operatorname{Arg}>\operatorname{Arg})\end{array}$ & $\begin{array}{l}\text { peripheral blood (38\%) } \\
\text { buccal mucosa (31\%) }\end{array}$ & $\begin{array}{l}\text { Sanger and } \\
\text { Pyrosequencing }\end{array}$ & + & assumed & $\begin{array}{l}\text { broad thumbs and } \\
\text { great toes }\end{array}$ & + \\
\hline Tajir et al. (2013) & one of the parents & $\begin{array}{l}\text { c.4361T>A } \\
\text { (p.Leu1454His) }\end{array}$ & - & $\begin{array}{l}\text { peripheral blood (0) buccal } \\
\text { mucosa }(0)\end{array}$ & Sanger & - & assumed & - & + \\
\hline $\begin{array}{l}\text { De Vries et al. } \\
\text { (2016) }\end{array}$ & one of the parents & $\begin{array}{l}\text { mos } \\
\text { c.5039_5041delCCT } \\
\text { (p.Ser1680del) }\end{array}$ & - & $\begin{array}{l}\text { peripheral blood (0) buccal } \\
\text { mucosa (ND) }\end{array}$ & $\begin{array}{l}\text { Sanger, RELP and } \\
\text { WES }\end{array}$ & + & unk & $\begin{array}{l}\text { RSTS and Filippi } \\
\text { syndrome }\end{array}$ & - \\
\hline Current study & mother & $\begin{array}{l}\text { c. } 3235 \mathrm{C}>\mathrm{T} \\
\left(\mathrm{p} . \mathrm{Gln} 1079^{*}\right)\end{array}$ & $\begin{array}{l}\operatorname{mos} c .3235 C>T \\
\left(p . G \ln 1079^{*}\right)\end{array}$ & $\begin{array}{l}\text { peripheral blood (3.64\%) } \\
\text { buccal mucosa (1.94\%) }\end{array}$ & high-depth NGS & + & assumed & RSTS & + \\
\hline
\end{tabular}

mos, mosaic; unk, unknown; ND, not determined; FISH, fluorescence in situ hybridization; RELP, restriction fragment length polymorphism analysis; WES, whole-exome sequencing; NGS, next-generation sequencing. 
further high-depth NGS detection in multiple specimens might be offered to identify potential low-level parental mosaicism in an effort to provide an assessment of recurrence risk in these families.

\section{DATA AVAILABILITY STATEMENT}

The supporting data for the current study are available from the corresponding authors upon reasonable request.

\section{ETHICS STATEMENT}

The studies involving human participants were reviewed and approved by Ethics Committee of The First Affiliated Hospital of Sun Yat-sen University. Written informed consent to participate in this study was provided by the participants' legal guardian/next of kin. Written informed consent was obtained from the individual(s), and minor(s)' legal guardian/next of kin, for the publication of any potentially identifiable images or data included in this article.

\section{REFERENCES}

Aarabi, M., Sniezek, O., Jiang, H., Saller, D. N., Bellissimo, D., Yatsenko, S. A., et al. (2018). Importance of complete phenotyping in prenatal whole exome sequencing. Hum. Genet. 137, 175-181. doi: 10.1007/s00439-017-1860-1

Bartsch, O., Kress, W., Kempf, O., Lechno, S., Haaf, T., and Zechner, U. (2010). Inheritance and variable expression in Rubinstein-Taybi syndrome. Am. J. Med. Genet. A 152A, 2254-2261. doi: 10.1002/ajmg.a.33598

Campbell, I. M., Stewart, J. R., James, R. A., Lupski, J. R., Stankiewicz, P., Olofsson, P., et al. (2014). Parent of origin, mosaicism, and recurrence risk: probabilistic modeling explains the broken symmetry of transmission genetics. Am. J. Hum. Genet. 95, 345-359. doi: 10.1016/j.ajhg.2014.08.010

Cao, Y., Tokita, M. J., Chen, E. S., Ghosh, R., Chen, T., Feng, Y., et al. (2019). A clinical survey of mosaic single nucleotide variants in disease-causing genes detected by exome sequencing. Genome Med. 11:48. doi: 10.1186/s13073-019-0658-2

Chiang, P. W., Lee, N. C., Chien, N., Hwu, W. L., Spector, E., and Tsai, A. C. (2009). Somatic and germ-line mosaicism in Rubinstein-Taybi syndrome. Am. J. Med. Genet. A 149A, 1463-1467. doi: 10.1002/ajmg.a.32948

De Vries, T. I., Monroe, G. R., Van Belzen, M. J., Van Der Lans, C. A., Savelberg, S. M., Newman, W. G., et al. (2016). Mosaic CREBBP mutation causes overlapping clinical features of Rubinstein-Taybi and Filippi syndromes. Eur. J. Hum. Genet. 24, 1363-1366. doi: 10.1038/ejhg.2016.14

Fu, F., Li, R., Lei, T. Y., Wang, D., Yang, X., Han, J., et al. (2020). Compound heterozygous mutation of the ASXL3 gene causes autosomal recessive congenital heart disease. Hum. Genet. 140, 333-348. doi: 10.1007/s00439-020-02200-Z

Gambin, T., Liu, Q., Karolak, J. A., Grochowski, C. M., Xie, N. G., Wu, L. R., et al. (2020). Low-level parental somatic mosaic SNVs in exomes from a large cohort of trios with diverse suspected Mendelian conditions. Genet. Med. 22, 1768-1776. doi: 10.1038/s41436-020-0897-z

Gervasini, C., Castronovo, P., Bentivegna, A., Mottadelli, F., Faravelli, F., Giovannucci-Uzielli, M. L., et al. (2007). High frequency of mosaic CREBBP deletions in Rubinstein-Taybi syndrome patients and mapping of somatic and germ-line breakpoints. Genomics 90, 567-573. doi: 10.1016/j.ygeno.2007.07.012

Hennekam, R. C. (2006). Rubinstein-Taybi syndrome. Eur. J. Hum. Genet. 14, 981-985. doi: 10.1038/sj.ejhg.5201594

Hennekam, R. C., Van Den Boogaard, M. J., Sibbles, B. J., and Van Spijker, H. G. (1990). Rubinstein-Taybi syndrome in The Netherlands. Am. J. Med. Genet. Suppl. 6, 17-29. doi: 10.1002/ajmg.1320370604

\section{AUTHOR CONTRIBUTIONS}

SL, YZ, and YL designed the study and wrote the manuscript. $\mathrm{ZH}, \mathrm{LH}$, and TL collected the clinical data and samples from the family. JL, JW, and $\mathrm{PH}$ performed the genetic analysis and evaluation of the variants. All authors contributed to the article and approved the submission.

\section{FUNDING}

This work was supported by the National Natural Science Foundation of China (82001564 and 81801705) and Natural Science Foundation of Guangdong Province, China (2018A030313832).

\section{ACKNOWLEDGMENTS}

We thank all the family members for their cooperation in this study.

Hu, P., Martinez, A. F., Kruszka, P., Berger, S., Roessler, E., and Muenke, M. (2019). Low-level parental mosaicism affects the recurrence risk of holoprosencephaly. Genet. Med. 21, 1015-1020. doi: 10.1038/s41436-018-0 261-8

Jonsson, H., Sulem, P., Arnadottir, G. A., Palsson, G., Eggertsson, H. P., Kristmundsdottir, S., et al. (2018). Multiple transmissions of de novo mutations in families. Nat. Genet. 50, 1674-1680. doi: 10.1038/s41588-018-0259-9

Kalkhoven, E., Roelfsema, J. H., Teunissen, H., Den Boer, A., Ariyurek, Y., Zantema, A., et al. (2003). Loss of CBP acetyltransferase activity by PHD finger mutations in Rubinstein-Taybi syndrome. Hum. Mol. Genet. 12, 441-450. doi: $10.1093 / \mathrm{hmg} / \mathrm{ddg} 039$

Korzus, E. (2017). Rubinstein-Taybi syndrome and epigenetic alterations. $A d v$. Exp. Med. Biol. 978, 39-62. doi: 10.1007/978-3-319-53889-1_3

Liu, A., Yang, X., Yang, X., Wu, Q., Zhang, J., Sun, D., et al. (2019). Mosaicism and incomplete penetrance of PCDH19 mutations. J. Med. Genet. 56, 81-88. doi: 10.1136/jmedgenet-2017-105235

Rodríguez-Martín, C., Robledo, C., Gómez-Mariano, G., Monzón, S., Sastre, A., Abelairas, J., et al. (2020). Frequency of low-level and high-level mosaicism in sporadic retinoblastoma: genotype-phenotype relationships. J. Hum. Genet. 65, 165-174. doi: 10.1038/s10038-019-0696-z

Smith, T. A., Yau, S. C., Bobrow, M., and Abbs, S. J. (1999). Identification and quantification of somatic mosaicism for a point mutation in a Duchenne muscular dystrophy family. J. Med. Genet. 36, 313-315.

Tajir, M., Fergelot, P., Lancelot, G., Elalaoui, S. C., Arveiler, B., Lacombe, D., et al. (2013). Germline mosaicism in Rubinstein-Taybi syndrome. Gene 518, 476-478. doi: 10.1016/j.gene.2012.12.105

Wiley, S., Swayne, S., Rubinstein, J. H., Lanphear, N. E., and Stevens, C. A. (2003). Rubinstein-Taybi syndrome medical guidelines. Am. J. Med. Genet. A 119A, 101-110. doi: 10.1002/ajmg.a.10009

Conflict of Interest: The authors declare that the research was conducted in the absence of any commercial or financial relationships that could be construed as a potential conflict of interest.

Copyright (c) 2021 Lin, He, Huang, Liu, Lei, Wu, Huang, Zhou and Luo. This is an open-access article distributed under the terms of the Creative Commons Attribution License (CC BY). The use, distribution or reproduction in other forums is permitted, provided the original author(s) and the copyright owner(s) are credited and that the original publication in this journal is cited, in accordance with accepted academic practice. No use, distribution or reproduction is permitted which does not comply with these terms. 